\title{
Microbiomes and Childhood Malnutrition: What Is the Evidence?
}

\author{
Michelle K. McGuire $^{a}$ Mark A. McGuire \\ aMargaret Ritchie School of Family and Consumer Sciences, University of Idaho, Moscow, ID, USA; \\ ${ }^{b}$ Department of Animal, Veterinary, and Food Sciences, University of Idaho, Moscow, ID, USA
}

\section{Key Messages}

- Healthy and malnourished infants have different fecal microbiomes, and this difference can precede onset of malnutrition suggesting that microbiome community structure might impart resilience and/or risk for malnutrition.

- Early differences in fecal microbiomes can be associated with later risk for overweight and obesity, but these differences vary by population and sometimes by sex.

- Longitudinal studies designed to prospectively assess variation among infant gastrointestinal microbiomes (and their likely sources, such as the human milk microbiome), infant feeding practices, nutritional status (including micronutrients), and systemic and intestinal inflammation are needed to tease apart these potentially important relationships.

\section{Keywords}

Growth · Health · Human milk · Infant · Malnutrition ·

Microbiome $\cdot$ Human nutrition · Obesity

\section{Abstract}

Both undernutrition and overnutrition continue to represent enduring global health crises, and with the growing implications of both forms of malnutrition occurring simultaneously in individuals and populations (referred to as the double burden of malnutrition), understanding their biological and environmental causes is a primary research and humanitarian necessity. There is growing evidence of a bidirectional association between variation in the gastrointestinal (GI) microbiome and risk of/resilience to malnutrition during early life. For example, studies of siblings who discordantly do or do not develop severe malnutrition show clear differences in the diversity and composition of fecal microbiomes. These differences are transiently lessened during refeeding but re-emerge thereafter. These findings have been somewhat recapitulated using animal models, but small sample sizes and limited range complicate interpretation of results and applicability to humans. Mechanisms driving these differences are currently unknown but likely involve a combination of inflammatory pathways (and perhaps antioxidant status of the host) and effects on nutrient availability, requirements, and utilization by both host and microbe. A less robust literature also suggests that variation in $\mathrm{Gl}$ microbiome is associated with risk for obesity during childhood. The putative impact of Gl microbiomes on malnutrition is likely modified by a variety of important variables such as genetics (likely driven, in part, by evolution), environmental pathogen exposure and its timing, dietary factors, and cultural/societal pattern (e.g., use of antibiotics). Given the growing double burden of malnutrition, this topic karger@karger.com

www.karger.com/anm (c) 2021 S. Karger AG, Basel

$$
\text { v'⿳亠丷厂 }
$$

Correspondence to:

Michelle K. McGuire, smcguire a uidaho.edu 
demands a focused interdisciplinary approach that expands from merely characterizing differences and longitudinal changes in fecal microbes to examining their functionality during early life. Understanding the complex composition of human milk and how its components impact establishment and maintenance of the recipient infant's GI microbiome will also undoubtedly shed important light on this topic.

(C) 2021 S. Karger AG, Basel

\section{Introduction}

Preventing and treating malnutrition, particularly in children, remain a global health priority [1]. Preventing undernutrition is important because inadequate food intake increases risks for nutritional deficiencies, impaired immune function, and delayed cognitive and physical development. Similarly, being overweight increases one's risk for a variety of diet-related noncommunicable diseases such as heart disease, stroke, diabetes, and cancer. Undernutrition and overnutrition have, in the past, been considered somewhat separate health challenges affecting different populations. However, with industrialization has come the simultaneous double burden of malnutrition (DBM), even within the same household or population [2]. Indeed, the prevalence of obesity continues to rise on a global basis while nutrient deficiencies remain common [2, 3].

Whereas the drivers of this somewhat new nutrition reality of DBM are clearly complex, malnutrition is often not as simple as nutrient deprivation or excess [4, 5]. For example, there is mounting evidence that a dysbiosis of enteric microbes might play a role in predisposing an individual to both extremes of the nutrient status continuum (see Table 1 for a summary of common measures of malnutrition in children). The opportunity to alter the enteric microbiome for optimizing lifelong health is likely greatest in the first 1,000 days of life [6], dovetailing nicely the concept of the developmental origins of health and disease ( $\mathrm{DOHaD})$, which proposes that variation in fetal and infant programming through environmental exposures impact lifelong health [7], and the hygiene hypothesis and related old friends hypothesis which posit that (1) early life exposure to microorganisms protects against allergic disease [8] and (2) early life exposure to microorganisms with which we have co-evolved is needed to develop tolerance so they do not evoke unhealthy inflammatory responses [9], respectively. Another important concept is eco-homeorhesis which posits that what is "normal" and "healthy" in 1 ecosystem may not confer optimal fitness in another [10]. Indeed, it is possible that there exists no 1-size-fits-all construct when it comes to a healthy GI microbiome in early life. Rather, as- sembly of an "optimal" Gl microbiome likely differs depending on a multitude of factors such as genetics, endemic microbes, chronic food availability, and cultural practices. Relationships among these concepts, nutrition, the enteric microbiome, and short- and long-term health are illustrated in Figure 1.

The focus of this study is to, largely through the lenses of these various overlapping concepts and hypotheses, review the complex interactions among early life nutrition, establishment and variation in Gl microbiomes, and overall health and nutritional status during childhood. We mainly discuss human studies of general malnutrition, but also briefly consider individual nutrients (particularly iron) and selected animal studies.

\section{Factors Impacting Early Establishment of GI Microbiome}

Whereas it has long been held that the healthy infant is born with a sterile Gl tract, some evidence suggests that initial microbial colonization may occur in utero [11, 12], although skepticism remains (e.g., [13]). Nonetheless, the origin of a large proportion (if not all) of the pioneering bacteria is related to exposure during birth. Azad and colleagues estimated that $70 \%$ of colonizing Gl bacteria originate from maternal feces in vaginally delivered infants, whereas a smaller proportion ( 40\%) originate from this source in infants born via Caesarean section [14]; whether the Caesarean section is planned or emergency as well as antibiotic use also appear to impact early colonization $[15,16]$. However, not all studies have reported an enduring impact of delivery mode on GI microbiome [17].

After initial colonization, feeding mode directs establishment and stabilization of the GI microbiome - likely driven by differences between the components found in human milk $(\mathrm{HM})$ and formula. The fecal microbiota of breastfed infants is not only different in composition than that of formula-fed infants, but it is also more stable and less diverse $[15,18]$. There are likely many reasons for these differences including HM oligosaccharides (HMOs) indigestible by human enzymes but selectively metabolized by microbes [19]. Even within exclusively breastfed infants, different HMO profiles have been associated with variation in infant fecal microbiomes [20, 21]. Because $\mathrm{HMO}$ profiles are largely driven by maternal genetics [22], it is possible that variation in HMOs and the resulting infant Gl microbiome represent eco-homeorhetic adjustments to "normal" to support optimal health in a particular environment and may also support the "old friends" hypothesis in that these microbial profiles nurture endemic, nonpathogenic bacterial taxa in such a way that they are tolerated by and perhaps even benefit the host. HMO concentrations and profiles 
Table 1. Common measures of malnutrition in children

\begin{tabular}{ll}
\hline Condition & Commonly used metric and/or sign \\
\hline Low birth weight, g & $<2,500$ \\
MAM & Weight-for-height $Z$ score between -2 and -3 without bilateral pitting edema \\
SAM & Weight-for-height $Z$ score $<-3$, or mid-upper arm circumference $<115 \mathrm{~mm}$, or bilateral pitting edema \\
Kwashiorkor & SAM with bilateral pitting edema \\
Marasmus & SAM without bilateral pitting edema \\
Underweight & Weight-for-age $Z$ score $<-2$ \\
Stunting & Height-for-age $Z$ score $<-2$ \\
Wasting & Weight-for-height $Z$ score $<-2$ \\
Overweight & Body mass index $Z$ score $>2$ or weight-for-length $Z$ score $>97$ th percentile \\
At risk of overweight & Weight-for-length $Z$ score $>85$ th percentile
\end{tabular}

MAM, moderate acute malnutrition; SAM, severe acute malnutrition.

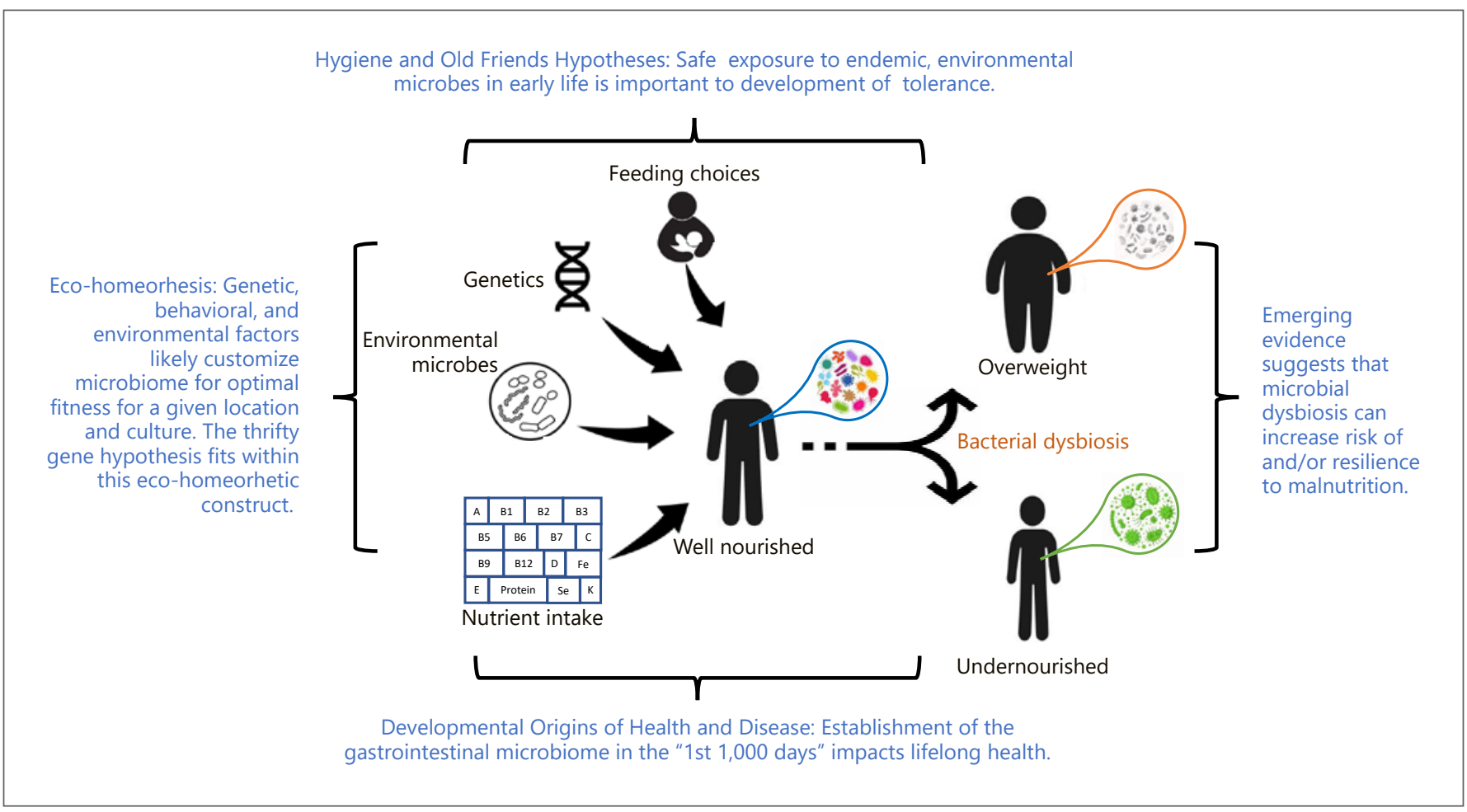

Fig. 1. Putative relationships among early-life microbial exposures, nutrition GI microbiomes, and malnutrition. Gl, gastrointestinal.

can also vary by season, suggesting that environment (e.g., food availability, pathogens) can impact their synthesis [23].

Many additional components (e.g., lysozyme and lactoferrin) in milk are known to affect GI microbes, but the presence of milk's own inherent microbiome is likely one of the most important [24]. Like HMO profiles [25], those of the HM microbiome (HMM) vary greatly among women and globally
[26]. Although milk and infant fecal microbiomes differ substantially in composition, variation in one is highly correlated with variation in the other $[27,28]$. The origins of the HMM are thought to be a combination of maternal skin and Gl tract, also fitting nicely into the old friend hypothesis and highlighting the likely importance of a tempered introduction of environmental microbes via breastfeeding/HM to develop toler- 
ance in the infant. It is also noteworthy that this microbial communication between mother and infant is likely bidirectional, with continuous inoculation of the mammary gland via the infant's mouth [29].

Introduction of complementary foods also impacts the infant's Gl microbiome [30, 31] although cessation of breastfeeding [32] might be more important suggesting that HM helps stabilize the infant's GI microbiome. Changes that occur around weaning are associated with shifts in the microbial community's capacity to metabolize typically consumed foods [32]. In addition, there are shifts in species that produce short-chain fatty acids and B vitamins (e.g., folate and vitamin $\mathrm{B}_{12}$ ), as well as taxa involved in amino acid metabolism [30, 33-35]. To our knowledge, there have been no studies designed to understand whether these longitudinal changes in Gl microbiomes and their functional capacities are customized to best suit the future dietary landscape, but this has been hypothesized [34].

In summary, many factors impact the diversity and composition of the Gl microbiome during early life, and there exists substantial variation in Gl microbiomes (and their functional capacities) among infants. Central to the aim of this manuscript, we ask whether this variability predisposes an infant to secondary malnutrition - be it undernutrition or overnutrition - and if so, is this a one-size-fits-all construct or is this effect modified by environment? Related, can nutritional status (e.g., iron) of an infant impact Gl microbiome to predispose the infant to acute and/or long-term health outcomes, and if so, is this relationship context specific?

\section{GI Microbiomes and Childhood Undernutrition}

It is well established that fecal microbiomes differ between lean and obese adults and that - although these differences are clearly due to a complex constellation of genetics, diet, and other biological and behavioral patterns - there might be particular Gl microbiomes that impart resilience to or risk of both under- and overnutrition. For instance, some taxa are genetically equipped to harvest a greater proportion of the energy in our foods than others, supplying volatile short-chained fatty acids that can serve as ATP

\section{Variation in early-life GI microbiome might also predispose an infant/child to malnutrition}

sources to enterocytes and colonocytes [36]. Individuals hosting a greater percentage of these taxa likely garner more calories from a given amount of food (example of the thrifty gene concept). Nonetheless, it is important to remember that

Infant Nutrition, Microbiomes, and Health only a small proportion ( 5-10\%) of energy obtained from the diet comes through microbial action in the large intestine. Rather, most is obtained via digestion by host enzymes and absorption in the stomach and small intestine. Some taxa can also synthesize essential vitamins (e.g., vitamin $B_{12}$ ), therefore supplying these nutrients to the host and possibly shielding him/her from deficiencies. While the absorption of vitamin $B_{12}$ synthesized in the colon or cecum is unlikely [37], some bacteria may benefit from increased levels. As such, variation in early-life Gl microbiome might also predispose an infant/ child to malnutrition and, conversely, variation in early life nutritional status may have acute and/or enduring impacts on the $\mathrm{Gl}$ microbiome. It is also possible that variation in the $\mathrm{Gl}$ microbiome plays a role in environmental enteric dysfunction, a subclinical and chronic disorder characterized by poor growth, blunting of the small intestine villi, nutrient malabsorption, and chronic inflammation [38].

To our knowledge, the first study comparing Gl microbiome between healthy and malnourished children was conducted by Monira et al. [39] and included 7 Bangladeshi children with low weight-for-height and 7 healthy children of study staff. There were substantial differences in both diversity and composition of the Gl microbiome: compared to the malnourished children, samples from healthy children had higher bacterial diversity and greater relative abundance of Bacteroidetes, whereas Klebsiella and Escherichia were 174and 9-fold lower. Interpretation, however, was severely limited by the fact that the study was small, cross-sectional, and the 2 cohorts were from different socioeconomic groups.

This study was followed by another [40] which compared Gl microbiome of Malawian twins discordant for kwashiorkor - a form of severe acute malnutrition (SAM) characterized by nutritional edema. Gl microbiomes of 9 healthy, same-gender twins were compared with those of 13 same-gender twin pairs who were discordant for kwashiorkor. Principal coordinates analysis was used to visualize composite variation in inferred fecal microbiome functionality with age. Overall metabolic functions of the healthy twin pairs' fecal microbiomes were found to steadily change over time as would be expected; the same was found for the healthy co-twins from discordant twin pairs. Conversely, metabolic functions of feces collected from children with kwashiorkor were age-delayed, and although they transiently became more like healthy children of similar ages during feeding this regressed when feeding was ceased. To assess causality, this research group then treated gnotobiotic mice 
with feces collected from twins in this study [41]. Results from mice (5 per single stool inoculum) in which transplantation efficiency was $>50 \%$ demonstrated reduced growth and lean mass in mice receiving stool from 5 stunted/underweight donors compared to mice receiving stool from 3 healthy donors. It is unclear if this reduction in replicates from the total donor population allowed for comparison between the mice inoculated with feces from twin cohorts or from a similar age cohort. A direct comparison within twin pairs would strengthen the confidence in the results. Further, transplant efficiency was defined based upon operational taxonomic unit (OTU) diversity relative to the uncultured donor sample, which suggests that relative abundance of bacterial taxa is not as important as mere presence in the community. Finally, clarity on food intake of the mice would be helpful in understanding the changes in growth. The authors

[41] report that food intake was not different for 4 days during the 35-day period, but it is not clear what days intake was measured. Random forest-based modeling identified Bifidobacterium longum in discriminating weight and lean mass gain when utilizing feces from children at 6 months of age while Faecalibacterium prausnitzii was more important when utilizing feces of children at 18 months of age. The potential impact of age-related $\mathrm{Gl}$ microbiome could be critical for potential success of interventions impacting malnutrition and emphasizing the importance of clear comparisons within an age group.

The same group then characterized what it considered to be healthy GI microbiome projections during the first 2 years of life in 12 healthy Bangladeshi infants and established a "microbiome-for-age $Z$ score" (MAZ) to assess Gl microbiome "maturity" [42]. This metric was applied to 64 Bangladeshi infants with SAM before and after nutritional treatment; SAM was associated with microbiome immaturity, and although MAZ score improved upon treatment, there was regression to immaturity afterward. In a subset of malnourished infants who provided samples before antibiotic administration (common to SAM treatment), lower microbial diversity prior to feeding was not attributable to antibiotics. The authors concluded that microbiome immaturity may play a role in predisposing young children to malnutrition. However, it should be noted that feeding mode and duration of exclusive breastfeeding were not controlled for, even though the authors provided some evidence that intake of infant formula was associated with higher maturity values. This weakness in experimental design makes its results difficult to interpret.
This study was followed by another that (1) assessed Gl microbiome in 27 twin pairs and 2 sets of triplets (from the same population) with healthy growth patterns, (2) compared findings with those published previously by Subramanian et al. [42] in Bangladesh (they were similar), (3) and related MAZ to subsequent growth in the Malawian cohort [43]. They found that MAZ at 12 months predicted future ponderal (but not linear) growth at 18 months, but again myriad confounding and possibly interacting factors (e.g., maternal nutritional status, variation in milk composition, and infant feeding practices) cannot be ruled out. In fact, lack of controlling for these and other confounding factors either in experimental design or statistical analysis is a major weakness of most of the research conducted on this topic and the reader will notice that this theme represents a common thread running through this study. A subsequent study [44] also suggested an expected maturation in the Gl virome among the Malawian twin cohort; this trajectory was impaired in SAM and not repaired with refeeding.

The researchers then used a defined 25-bacterium community isolated from a single fecal sample collected from 1 stunted Malawian infant transplanted into germ-free mice fed a diet representative of that which is consumed in Malawi to test whether the addition of bovine milk oligosaccharides (BMOs) could rescue growth [43]. Mice (4-5 per group) fed the BMO-fortified diet had increased body weight compared to mice fed the control diet with or without inulin. Unfortunately, comparison to mice transplanted with microbiota present in healthy Malawi infants was absent in the experimental design. Mice not fed BMO had sustained body weight losses for 30 days after colonization differing from the work of Blanton et al. [41] where only temporary body weight $(<8$ days) losses were noted in any group. Further, while food consumption was not statistically different, the mice fed the BMO-fortified diet consumed $25 \%$ more than controls. Similar effects were found with gnotobiotic piglets. Additional studies utilizing larger sample sizes and appropriate controls are needed to further understand these findings.

It is interesting to speculate what causes or prevents "maturation" of the fecal microbiota, but Vonaesch and colleagues [45] provide strong evidence that stunted children may have small intestine bacterial overgrowth dominated by taxa typically found in the oropharyngeal cavity. Their data suggest a "decompartmentalization" of the Gl tract and are not only interesting from a mechanistic point of view but also remind us that microbial communities vary along the Gl tract, and that 
the fecal microbiome likely provides a poor representation of what is likely more important for nutrition, that is, the small intestine microbiome.

In another related study utilizing data from the Bangladeshi and Malawi cohorts described above, Gough and colleagues [46] used a case-control approach to investigate whether particular fecal microbial community structures or individual taxa are associated with linear growth faltering. A total of 10 cases and 8 controls from Malawi, and 6 cases and 5 controls from Bangladesh, where cases were defined as $\mathrm{HAZ} \leq-3$ and controls as $-3<H A Z \leq-2$. They found that reduced fecal microbiome diversity and increased relative abundance of Acidaminococcus sp. predicted more severe later growth deficits and hypothesized that microbial fermentation of glutamate might play a role in this association. To test this, they used KEGG enzyme abundance data for the Malawi cohort and found that the abundance of genes encoding key glutamatemetabolizing enzymes was negatively associated with future height-for-age $Z$ score. However, cases in both cohorts were younger and relatively shorter (HAZ) than controls making it impossible to disentangle whether the differences in fecal microbiome are related to age-related factors (e.g., exclusive breastfeeding status) or health status. In addition, because the controls were also stunted, the data cannot be used to infer differences between well growing and growth-stunted infants. Future studies should better account for these important variables.

In a set of studies carried out in Senegal and Niger, Million and colleagues [47] studied 69 children with varying nutritional status. Location was found to be the major driver of overall fecal microbial community structure when all children were considered together, whereas age, sex, and nutritional status did not explain much of the overall taxonomic variation. However, the researchers found lower levels of obligate anaerobes associated with malnutrition. This approach was followed up with an individual patient data meta-analysis which included 108 children with SAM and 77 well-nourished children from 5 African and Asian countries. Results indicate that members of the Bacteroidaceae, Eubacteriaceae, Lachnospiraceae, and Ruminococceae families were depleted while Enterococcus faecalis, Escherichia coli, and Staphylococcus aureus were consistently more abundant in feces collected from children with SAM. Depletion of obligate anaerobes, decreased total bacterial number, and lower relative abundance of Methanobrevibacter smithii (a member of the Archaea domain) were also associated with malnutrition. M. smithii is thought to enhance Gl bacterial polysaccharide digestion by optimizing hydrogen levels; its potential role in increasing metabolizable energy has been discussed as has its use as a therapeutic for modulating energy balance in humans [48].
The authors suggest that maternal antioxidant intake (and thus, milk antioxidant composition) might modify the importance of fecal redox capacity to infant nutritional status. If correct, then it is likely that the presence of Gl microbiota with redox capacity might be particularly important in cases of maternal antioxidant deficiency and/or nonexclusive breastfeeding and inadequate infant antioxidant consumption - again, suggesting that what would be considered "optimal" microbiomes in various contexts differs.

\section{Maternal antioxidant intake (and thus, milk antioxidant composition) might modify the importance of fecal redox capacity to infant nutritional status}

In a subsequent study [49], the same group compared Gl microbiomes of 5 healthy infants and 10 infants with kwashiorkor; they also found a depletion in oxygen-sensitive prokaryotes including M. smithii. Diversity of anaerobic taxa was lower in the malnourished infants, and much of this difference was due to species not previously identified in human stool samples. The authors posited that there is a "loss" of anaerobic species in patients with kwashiorkor. It is worth noting, however, that although ages were not statistically different between the groups the malnourished cohort was substantially younger than the controls ( 13.4 vs. 25.1 months). As such, differences could be explained by myriad other factors such as breastfeeding status. In addition, the small sample sizes combined with the fact that subjects were recruited from 2 distinct locations (Senegal and Niger) make the results difficult to interpret. Future studies should endeavor to recruit healthy and malnourished subjects at similar ages living in the same location.

In the only study to our knowledge to compare infants with kwashiorkor ( $n=54$ ) to those with marasmus ( $n=33$ ), Kristensen and colleagues [50] studied Ugandan children aged 6-24 months. After adjusting for sex and diarrhea at admission, they found higher diversity in samples collected from infants with kwashiorkor. When severity of wasting, mid-upper arm circumference, and stunting were considered separate from type of SAM, no relationships were found with overall Gl microbiome. There were also no differences in individual taxa. Unfortunately, markers of inflammation and 
differences in microbial community function were not assessed. In addition, children with kwashiorkor were older than those with marasmus (17 vs. 15 months, respectively; $p=$ 0.04), and age and infant feeding patterns were not controlled for in the analyses. This is particularly unfortunate given the fact that these authors had previously published findings [51] that children with kwashiorkor in this population were less likely to be breastfed and that household dietary diversity scores were also lower in these children.

In contrast, in a longitudinal study of 78 Peruvian infants between 5 and 12 months of age [52], those who became stunted during the study had higher bacterial diversity and altered Gl microbiome coupled with elevated biomarkers of inflammation that preceded growth faltering. Differences in fecal microbial diversity also preceded growth faltering. Importantly, the researchers took care to document infant feeding practices and reported no differences in duration of exclusive breastfeeding and age at introduction of solid foods; this represents an important strength of this study compared to almost all others on the topic. The authors concluded that the origin of immune activation in stunted children may be sustained, low-grade microbial translocation across the Gl mucosa. However, because differences in Gl microbiome were also present prior to growth stunting it is also possible that microbially derived inflammatory metabolites might drive chronic local irritation. Metagenomic analysis to examine microbial function would be helpful to tease apart these intertwining possibilities.

In another study [53] characterizing longitudinal relationships among GI microbiome, growth, and inflammation in 691 Malawian infants, MAZ and GI microbial diversity were associated with weight gain from 6 to 12 months, but associations with systemic inflammation were inconsistent. Although infant feeding status was not reported, the analyses were adjusted for infant age on day of fecal collection. Other studies (e.g., [54]), have also provided weak evidence for a link between an "inflammatory" Gl microbiome and growth, but these studies lack evaluation of inflammatory status in the infant.

\section{GI Microbiome Functionality and Childhood Undernutrition}

Highlighting the potential importance of Gl microbiome function rather than composition, Mayneris-Perxachs et al. [55] utilized a case-control study in malnourished versus wellnourished Brazilian infants $(n=326) 6-24$ months of age. Importantly, cases $(n=158)$ and controls $(n=168)$ were matched for age, although matching was within 6 months - a relatively long period of time in this context - and cases were older than controls (14.8 vs. 12.3 months, $p<0.001$ ). In addition, birth weight was lower in cases than controls ( -1.7 vs. -0.52 WAZ, $p<0.001)$. Whether the infant was breastfed was apparently similar between groups, although it is unclear from the report if this refers to ever having been breastfed or breastfed at the time of sample/data collection. Whereas they did not directly assess Gl microbiome, they evaluated urinary metabolic phenotypes and indirectly inferred source (host vs. microbe). They found that both stunting and wasting were associated with increased proteolytic activity of the infants' Gl microbiomes as well as dysregulated choline metabolism and metabolic adaptations to reduce energy expenditure. Accelerated catch-up growth was seen in children with the most pronounced energy-saving metabolic adaptations. The authors posit that alterations in methyl donor capacity might trigger early-life epigenetic programming toward a "thrifty" phenotype. Whereas a favoring of Gl microbiota that is able to utilize protein as an energy source seems like a reasonable and complementary response to inadequate nutrient availability, it remains unclear how this would occur. Also, will increased proteolytic activity reduce availability of essential amino acids to the infant and lead to decreased growth and synthesis of related compounds such as serotonin? Answering these questions will require studies coupling longitudinal assessment of infant growth, Gl microbiomes and metabolites, possibly stably labelled amino acids, and circulating metabolomics.

To help in this regard, Giallourou et al. [56] evaluated longitudinally collected urine samples and growth in malnourished Peruvian, Bangladeshi, and Tanzanian infants ( $n=281$, 249 , and 249, respectively) during the first 24 months of life. Infants from each location ( $n=22,28$, and 13 for Peru, Bangladesh, and Tanzania, respectively) with $L A Z \geq-0.75$ at birth and $\geq-1.25$ at 24 months of age were classified as a "healthy" reference group. Both plasma and urinary metabolomes were assessed, with the latter (in healthy infants) being used to explore the existence of age-discriminatory metabolites common across cohorts (phenome-for-age $Z$ score, PAZ). PAZ scores in malnourished children were lower than those of their healthy counterparts, and low PAZ predicted later linear and ponderal growth faltering. After controlling for cofounders such as breastfeeding and diarrhea (none of which were found to be important), several urinary metabolites arising from microbial-host co-metabolism were positively correlated with age and/or nutritional status - many related to protein and amino acid metabolism. Importantly, these generalized findings were consistent among locations suggesting that they might represent relationships that might be applicable to other populations. Whether these associations between PAZ 
and nutritional status are causal in nature is unknown, as is whether differences in Gl microbiomes represent mediating factors.

\section{Micronutrients (Particularly Iron), Microbiomes, and Malnutrition}

Although most research regarding Gl microbiome and earlylife malnutrition deals with generalized undernutrition (e.g. kwashiorkor), micronutrient intake might also be important. Although many microbes synthesize vitamins that can be used both by themselves and their hosts, they also rely on the presence of some nutrients (e.g., some vitamins, essential minerals) for survival. It is plausible, therefore, that endemic nutrient deficiencies might evolutionarily select for Gl microbes that produce those nutrients - another possible example of eco-homeorhesis. Yet, aside from iron, very little research has been conducted on this topic resulting in a weak knowledge base in this regard. As such, here we focus mainly on studies relating infant iron consumption and status on the Gl microbiome.

\section{Endemic nutrient deficiencies might evolutionarily select for GI microbes that produce those nutrients}

In a double-blind, controlled intervention trial carried out in Côte d'Ivoire, iron supplementation of 6- to 14-yearold children ( $n=139$ ) increased fecal enterobacteria and decreased lactobacilli and bifidobacteria [57]. This shift was accompanied by increased calprotectin, a marker of GI inflammation. Additional negative outcomes of iron supplementation were reported in 6-month-old Kenyan infants ( $n=115$ ) [58] and 1-year-old Peruvian infants ( $n=95$ ) [59], with the latter study revealing sex-specific responses. These findings are bolstered by an extensive literature relating iron status (and supplementation) to risk of infection, particularly by pathogens with substantial requirements for this mineral - the most studied being those that cause malaria [60]. Indeed, Plasmodium falciparum has evolved elaborate mechanisms to sequester iron, and supplementing the host with iron can enhance this pathogen's survival and reproduction. This finding has led the WHO to recommend that iron interventions only be administered in areas of endemic malaria when diagnosis, prevention, and treatment schemes are employed [61].

Because most microbes have a requirement for iron, humans have evolved a constellation of mechanisms whereby iron is withheld from pathogens during infection, ultimately impairing bacterial growth while increasing risk for host iron deficiency. Interestingly, HM is a poor source of iron, and its iron content is relatively refractory to maternal iron status suggesting an adaptive advantage to low levels of iron consumption in early life. Several studies have provided evidence that, in addition to iron supplementation increasing risk for certain infections including those causing diarrhea, it can also impact the Gl microbiome of children. For instance, several have found that iron interventions increase Enterobacteriaceae and decrease Lactobacilli and Bifidobacteria [57, 58, 62, 63]. This finding is particularly interesting because members of the Lactobacillaceae family, common in milk and dairy products and considered to be health-promoting probiotics, have an extremely low requirement for iron. As such, when iron is abundant in the environment, these taxa are likely outcompeted by iron-requiring taxa. It is noteworthy that the impact of iron supplementation on Gl microbiome appears to be context specific, likely impacted by typical core Gl microbial community structures, chronic dietary intake, and endemic pathogen exposure. For example, Dostal and colleagues [64] found no effect of high-dose iron supplements on fecal microbiomes in iron-deficient children with low enteropathogen burden living in South Africa. This discrepancy in findings across locations highlights the need to study these interactions in each location of interest and the critical importance of not extending findings and conclusions drawn from one locale to another.

\section{GI Microbiomes and Childhood Obesity}

There is also substantial interest in the complex interactions among Gl microbiome, obesity risk, and inflammation in early life. One of the first studies to examine this was a casecontrol design in Finland; 24 normal-weight children were matched for gestational age and BMI at birth, delivery mode, probiotic intervention, duration of breastfeeding, and antibiotic use [65]. GI microbiome was assessed at 6 and 12 months and relationship to risk for obesity during the first 7 years of life assessed. Bifidobacterial numbers in stool samples during infancy were higher in children who remained healthy weight than those who became overweight; $S$. aureus was higher in those who became overweight. The researchers hypothesized that $S$. aureus may trigger low-grade inflammation, contributing to the development of obesity and link their findings 
to an extended concept of the hygiene hypothesis. In a companion case-control study involving 15 overweight children at 10 years of age and 15 healthy-weight children matched for sex, gestational age and BMI at birth, mode of delivery, and duration of breastfeeding, Luoto et al. [66] found that bifidobacterial numbers tended to be lower in fecal samples collected at 3 months of age in the overweight children. The same research team later reported that Staphylococcus was elevated in feces of infants' overweight mothers compared to healthy-weight mothers during pregnancy [67], supporting the possibility that the infants' fecal microbiomes were shaped, at least in part, either during delivery, via their mothers' HMM, or through other physical contacts in the shared environment. Evidence of intergenerational transmission of the overweight phenotype from mother to offspring via microbiome transfer has also been provided by Tun and colleagues [68], who utilized multiple mediator path modeling to show that infant fecal microbiome (particularly Lachnospiraceae) mediated the association between maternal prepregnancy overweight and risk of overweight in children at 1 and 3 years of age.

In a larger study of 330 healthy Danish infants, Bergstrom and colleagues [69] found that increasing abundance of several taxa (e.g., Firmicutes) and decreasing levels of $M$. smithii in feces of infants between 9 and 18 months of age were associated with greater BMI gains - a finding somewhat in conflict with that reported in malnourished children and described previously suggesting that $M$. smithii might facilitate microbial fermentation of polysaccharides in the colon. The reason for this discrepancy is unknown, but again highlights the importance of not assuming similar findings in disparate locations. Results from the CHILD study in Canada suggested that higher microbial richness and enrichment of Lachnospiraceae at 3-4 months increased risk of obesity at 12 months [70]. In a companion study [68], abundance of Lachnospiraceae at 3-4 months was associated with more frequent use of household disinfectants, and was found to statistically mediate an apparent association between disinfectant use and increased odds of becoming overweight or obese at 3 years. However, exclusive breastfeeding was associated with lower use of disinfectant, complicating interpretation. Dogra et al. [71] reported that infants who acquire higher levels of Bifidobacterium relatively later had lower adiposity at 18 months, but differences in feeding practices were not accounted for.

Several studies have also considered this topic in premature infants. Scheepers et al. [72] studied 909 preterm infants and found that higher levels of fecal $B$. fragilis at 1 month was associated with higher BMI between 1 and 10 years, but only when the infants consumed a low-fiber diet. This association was modified by maternal lifestyle factors, making the results challenging to interpret. Importantly, feeding practices in the first month (exclusive breastfeeding, formula feeding, or a combination) and duration of breastfeeding were controlled for in the analysis. White and colleagues [73] found that early detection of Bacteroides in stool samples from 218 preterm and term infants at 1 month was associated with reduced growth in the first 6 months in male infants; although the authors controlled for whether or not the infant had received formula they did not control for duration of breastfeeding. Additional evidence exists that the association between $\mathrm{Gl}$ microbiome and growth might be sex-specific [69, 74, 75].

It should be noted that, similar to the literature on antibiotics and growth faltering, there is a relatively large literature examining the association between antibiotic use in infancy and risk of developing obesity. Findings from these studies, however, are notably inconsistent [75-77]. Again, it is likely that confounders (e.g., infant Gl microbiome community structure and feeding patterns) modify any effect that might exist and that any impacts are geographically/culturally specific.

\section{Conclusions and Major Research Gaps}

Risk of and resilience to malnutrition as they relate to variation in the early-life GIM warrants continued examination because it is likely that, at least in some contexts, different GI microbiomes predispose (or program) individuals to acute and chronic health trajectories (hygiene and old friends hypotheses, DOHaD, 1st 1,000 days emphasis). Most studies to date are understandably epidemiologic in nature, and the design of most interventions has focused on describing members of the fecal microbiome that could impact health through inflammation and nutrient utilization. However, consumption and absorption of nutrients by the host and production and use of nutrients by bacteria can create competitive scenarios that are typically not considered in any of these studies. Importantly, what is a "healthy" Gl microbiome might be modified by genetics, nutrient availability, endemic microbe exposures, feeding patterns, and sex (eco-homeorhesis), but these interactions have not been studied using adequate prospective, longitudinal experimental designs in at-risk populations. Because Gl microbiomes are clearly different across locations and these customized differences might be important for acute and chronic health, researchers and public health experts should apply a healthy dose of restraint when considering extending findings from 1 location to another. In summary, the current state of the science is not adequate to conclude that variation in the early-life microbiome can lead to either undernutrition or obesity, and additional rigorous studies are needed in this regard. 
Research in this area must carefully take into account the age and feeding history of study participants, or these important factors might completely confound research results and substantially complicate their interpretation. When possible, studies should be conducted in more than one location so that we can better understand microbe-health relationships that are specific to one culture/region versus those that are common to all. Scientific focus should expand well beyond characterizing the association between generalized proteinenergy malnutrition, impaired growth, and energy imbalance (e.g., kwashiorkor and marasmus) and Gl microbiome. Rather, and as highlighted by evidence that iron supplementation during infancy and early childhood might negatively impact early-life Gl microbiome, micronutrients should also be considered. And, as always, studies should be powered sufficiently to address the major hypotheses being tested.

Elegant and complex animal studies have examined the effect of Gl microbiome through inoculation from healthy or stunted infants. These studies have examined an array of -omics to identify potential markers or causative factors for the phenotypes observed in the afflicted children. However, relatively poor establishment of the bacterial community into the germ-free models and limited numbers of replicates, both from donors and recipient animals within a donor cohort, restrict our ability to interpret the results - let alone apply them to the human condition. In addition, a lack of growth or even body weight loss with the limited animal numbers is a concern for validity of these models. Finally, these models which utilize weaned animals do not include the potential contribution of

\section{References}

1 Black MM, Lutter CK, Trude ACB. All children surviving and thriving: re-envisioning UNICEF's conceptual framework of malnutrition. Lancet Glob Health. 2020;8(6):e766-7.

2 Popkin BM, Corvalan C, Grummer-Strawn LM. Dynamics of the double burden of malnutrition and the changing nutrition reality. Lancet. 2020;395(10217):65-74.

3 United Nations Children's Fund (UNICEF), World Health Organization, International Bank for Reconstruction and Development/The World Bank. Levels and trends in child malnutrition: key findings of the 2020 edition of the joint child malnutrition estimates. Geneva: World Health Organization; 2020.

4 Prendergast AJ, Humphrey $\mathrm{JH}$. The stunting syndrome in developing countries. Paediatr Int Child Health. 2014;34(4):250-65.

5 Martorell R, Zongrone A. Intergenerational influences on child growth and undernutrition. Paediatr Perinat Epidemiol. 2012; 26(Suppl 1):302-14.

6 Robertson RC, Manges AR, Finlay BB, Prendergast AJ. The human microbiome and child growth - first 1000 days and beyond. Trends Microbiol. 2019;27(2):131-47.
HM to the nutrient or health status of children, which would be the normal feeding for the children studied. Thus, these models fail to reflect the various food sources consumed by children.

Given the growing double burden of malnutrition, understanding the complex relationships among infant nutrition, nutritional status, and microbiomes should garner intense research activity and funding. Adequately describing this complicated interaction will demand a focused interdisciplinary approach among nutritionists, microbiologists, immunologists, epidemiologists, and clinicians. For these studies to provide insight, they should expand from simply cataloging microbes to examining their functionality. Because HM contains a constellation of components (e.g., antimicrobial factors, nutrients, and microbes) that can impact establishment of the Gl microbiome, understanding these biological compounds and microorganisms is likely crucial to this type of research. The expanded use of well-powered, mechanistic studies using validated animal models is also clearly needed.

\section{Conflict of Interest Statement}

The writing of this article was supported by Nestle Nutrition Institute and the authors declare no other conflicts of interest.

\section{Author Contributions}

Both authors contributed equally to the writing of this manuscript.
7 Stiemsma LT, Michels KB. The role of the microbiome in the developmental origins of health and disease. Pediatrics. 2018;141(4): e20172437.

8 Sicherer SH, Sampson HA. Food allergy: a review and update on epidemiology, pathogenesis, diagnosis, prevention, and management. J Allergy Clin Immunol. 2018;141:41-58.

9 Rook GA, Raison CL, Lowry CA. Microbial "old friends", immunoregulation and socioeconomic status. Clin Exp Immunol. 2014; 177(1):1-12.

10 McGuire MK, Meehan CL, Brooker SA, Williams JE, Foster JA, McGuire MA. An evolutionary, biosocial perspective on variation in human milk microbes and oligosaccharides: An example of ecohomeorhesis? In: McGuire MK, McGuire MA, Bode L, editors. Prebiotics and probiotics in human milk. Elsevier Publishing; 2017.

11 Wilczyska P, Skaryska E, Lisowska-Myjak B. Meconium microbiome as a new source of information about long-term health and disease: questions and answers. J Matern Fetal Neonatal Med. 2019;32(4):681-6. 
12 Collado MC, Isolauri E, Laitinen K, Salminen S. Distinct composition of gut microbiota during pregnancy in overweight and normal-weight women. Am J Clin Nutr. 2008;88(4):894-9.

13 Perez-Muñoz ME, Arrieta MC, Ramer-Tait AE, Walter J. A critical assessment of the "sterile womb" and "in utero colonization" hypotheses: implications for research on the pioneer infant microbiome. Microbiome. 2017;5(1):48.

14 Azad MB, Konya T, Persaud RR, Guttman DS, Chari RS, Field CJ, et al. Child study investigators. Impact of maternal intrapartum antibiotics, method of birth and breastfeeding on gut microbiota during the first year of life: a prospective cohort study. BJOG. 2016; 123(6):983-93.

15 Azad MB, Konya T, Maughan H, Guttman DS, Field CJ, Chari RS, et al. Child study investigators. Gut microbiota of healthy Canadian infants: profiles by mode of delivery and infant diet at 4 months. CMAJ. 2013;185(5):385-94.

16 Langdon A, Crook N, Dantas G. The effects of antibiotics on the microbiome throughout development and alternative approaches for therapeutic modulation. Genome Med. 2016;8(1):39.

17 Chu DM, Ma J, Prince AL, Antony KM, Seferovic MD, Aagaard KM. Maturation of the infant microbiome community structure and function across multiple body sites and in relation to mode of delivery. Nat Med. 2017;23(3):314-26.

18 Fan W, Huo G, Li X, Yang L, Duan C, Wang T, et al. Diversity of the intestinal microbiota in different patterns of feeding infants by IIlumina high-throughput sequencing. World J Microbiol Biotechnol. 2013;29(12):2365-72.

19 Vandenplas Y, Carnielli VP, Ksiazyk J, Luna MS, Migacheva N, Mosselmans JM, et al. Factors affecting early-life intestinal microbiota development. Nutrition. 2020;78:110812.

20 Lewis ZT, Totten SM, Smilowitz JT, Popovic M, Parker E, Lemay $D G$, et al. Maternal fucosyltransferase 2 status affects the gut bifidobacterial communities of breastfed infants. Microbiome. 2015; 3:13.

21 Smith-Brown P, Morrison M, Krause L, Davies PS. Mothers secretor status affects development of children's microbiota composition and function: a pilot study. PLoS One. 2016;11(9):e0161211.

22 Williams JE, McGuire MK, Meehan CL, McGuire MA, Brooker SL, Kamau-Mbuthia EW, et al. Key genetic variants associated with variation of milk oligosaccharides from diverse human populations. Genomics. 2021;113(4):1867-75.

23 Davis JC, Lewis ZT, Krishnan S, Bernstein RM, Moore SE, Prentice AM, et al. Growth and morbidity of gambian infants are influenced by maternal milk oligosaccharides and infant gut microbiota. Sci Rep. 2017;7:40466.

24 Pace RM, Williams JE, Robertson B, Lackey KA, Meehan CL, Price $W J$, et al. Variation in human milk composition is related to differences in milk and infant fecal microbial communities. Microorganisms. 2021;9(6):1153.

25 McGuire MK, Meehan CL, McGuire MA, Williams JE, Foster J, Sellen DW, et al. What's normal? Oligosaccharide concentrations and profiles in milk produced by healthy women vary geographically. Am J Clin Nutr. 2017:105(5):1086-100.
26 Lackey KA, Williams JE, Meehan CL, Zachek JA, Benda ED, Price WJ, et al. What's normal? Microbiomes in human milk and infant feces are related to each other but vary geographically: the inspire study. Front Nutr. 2019;6:45.

27 Pannaraj PS, Li F, Cerini C, Bender JM, Yang S, Rollie A, et al. Association between breast milk bacterial communities and establishment and development of the infant gut microbiome. JAMA Pediatr. 2017;171(7):647-54.

28 Williams JE, Carrothers JM, Lackey KA, Beatty NF, Brooker SL, Peterson $\mathrm{HK}$, et al. Strong multivariate relations exist among milk, oral, and fecal microbiomes in mother-infant dyads during the first six months postpartum. J Nutr. 2019;149(6):902-14

29 Ramsay DT, Mitoulas LR, Kent JC, Larsson M, Hartmann PE. The use of ultrasound to characterize milk ejection in women using an electric breast pump. J Hum Lact. 2005;21(4):421-8.

30 Koenig JE, Spor A, Scalfone N, Fricker AD, Stombaugh J, Knight R, et al. Succession of microbial consortia in the developing infant gut microbiome. Proc Natl Acad Sci USA. 2011;108(Suppl 1):457885.

31 Palmer C, Bik EM, DiGiulio DB, Relman DA, Brown PO. Development of the human infant intestinal microbiota. PLoS Biol. 2007: 5(7):e177.

32 Bäckhed F, Roswall J, Peng Y, Feng Q, Jia H, Kovatcheva-Datchary $P$, et al. Dynamics and stabilization of the human gut microbiome during the first year of life. Cell Host Microbe. 2015;17(5):690703.

33 Tannock GW, Lawley B, Munro K, Gowri Pathmanathan S, Zhou SJ, Makrides M, et al. Comparison of the compositions of the stool microbiotas of infants fed goat milk formula, cow milk-based formula, or breast milk. Appl Environ Microbiol. 2013;79(9):3040-8.

34 Yatsunenko T, Rey FE, Manary MJ, Trehan I, Dominguez-Bello MG, Contreras $\mathrm{M}$, et al. Human gut microbiome viewed across age and geography. Nature. 2012;486(7402):222-7.

35 Vallès Y, Artacho A, Pascual-García A, Ferrús ML, Gosalbes MJ, Abellán JJ, et al. Microbial succession in the gut: directional trends of taxonomic and functional change in a birth cohort of Spanish infants. PLoS Genet. 2014;10(6):e1004406.

36 Holmes ZC, Silverman JD, Dressman HK, Wei Z, Dallow EP, Armstrong SC, et al. Short-chain fatty acid production by gut microbiota from children with obesity differs according to prebiotic choice and bacterial community composition. mBio. 2020;11(4): e00914-20.

37 Schjønsby H. Vitamin B12 absorption and malabsorption. Gut. 1989;30:1686-91.

38 Bartelt LA, Bolick DT, Guerrant RL. Disentangling microbial mediators of malnutrition: modeling environmental enteric dysfunction. Cell Mol Gastroenterol Hepatol. 2019;7(3):692-707.

39 Monira S, Nakamura S, Gotoh K, Izutsu K, Watanabe H, Alam NH, et al. Gut microbiota of healthy and malnourished children in Bangladesh. Front Microbiol. 2011;2:228.

40 Smith MI, Yatsunenko T, Manary MJ, Trehan I, Mkakosya R, Cheng J, et al. Gut microbiomes of Malawian twin pairs discordant for kwashiorkor. Science. 2013;339(6119):548-54. 
41 Blanton LV, Charbonneau MR, Salih T, Barratt MJ, Venkatesh S, Ilkaveya $O$, et al. Gut bacteria that prevent growth impairments transmitted by microbiota from malnourished children. Science. 2016 Feb 19;351(6275):10.

42 Subramanian S, Huq S, Yatsunenko T, Haque R, Mahfuz M, Alam MA, et al. Persistent gut microbiota immaturity in malnourished Bangladeshi children. Nature. 2014;510(7505):417-21.

43 Charbonneau MR, Blanton LV, DiGiulio DB, Relman DA, Lebrilla CB, Mills DA, et al. A microbial perspective of human developmental biology. Nature. 2016;535(7610):48-55.

44 Reyes A, Blanton LV, Cao S, Zhao G, Manary M, Trehan I, et al. Gut DNA viromes of Malawian twins discordant for severe acute malnutrition. Proc Natl Acad Sci USA. 2015;112(38):11941-6.

45 Vonaesch P, Morien E, Andrianonimiadana L, Sanke H, Mbecko JR, Huus KE, et al. Afribiota investigators: stunted childhood growth is associated with decompartmentalization of the gastrointestinal tract and overgrowth of oropharyngeal taxa. Proc Natl Acad Sci USA. 2018;115(36):E8489.

46 Gough EK, Stephens DA, Moodie EE, Prendergast AJ, Stoltzfus RJ, Humphrey $\mathrm{JH}$, et al. Linear growth faltering in infants is associated with Acidaminococcus sp. and community-level changes in the gut microbiota. Microbiome. 2015;3:24.

47 Million M, Tidjani Alou M, Khelaifia S, Bachar D, Lagier JC, Dione $\mathrm{N}$, et al. Increased gut redox and depletion of anaerobic and methanogenic prokaryotes in severe acute malnutrition. Sci Rep. 2016; $6: 26051$

48 Samuel BS, Hansen EE, Manchester JK, Coutinho PM, Henrissat B, Fulton R, et al. Genomic and metabolic adaptations of Methanobrevibacter smithii to the human gut. Proc Natl Acad Sci USA. 2007:104(25):10643-8.

49 Tidjani Alou M, Million M, Traore SI, Mouelhi D, Khelaifia S, Bachar $D$, et al. Gut bacteria missing in severe acute malnutrition, can we identify potential probiotics by culturomics? Front Microbiol. 2017;8:899.

50 Kristensen KH, Wiese M, Rytter MJ, Özçam M, Hansen LH, Namusoke $\mathrm{H}$, et al. Gut microbiota in children hospitalized with oedematous and non-oedematous severe acute malnutrition in Uganda. PLoS Negl Trop Dis. 2016;10(1):e0004369.

51 Rytter MJ, Namusoke H, Babirekere-Iriso E, Kæstel P, Girma T, Christensen VB, et al. Dietary and clinical correlates of oedema in children with severe acute malnutrition: a cross-sectional study. BMC Pediatr. 2015;15:25.

52 Zambruni M, Ochoa TJ, Somasunderam A, Cabada MM, Morales $M L$, Mitreva $M$, et al. Stunting is preceded by intestinal mucosal damage and microbiome changes and is associated with systemic inflammation in a cohort of Peruvian infants. Am J Trop Med Hyg. 2019;101(5):1009-17.

53 Kamng'ona AW, Young R, Arnold CD, Kortekangas E, Patson N, Jorgensen JM, et al. The association of gut microbiota characteristics in Malawian infants with growth and inflammation. Sci Rep. 2019; 9(1):12893.

54 Dinh DM, Ramadass B, Kattula D, Sarkar R, Braunstein P, Tai A, et al. Longitudinal analysis of the intestinal microbiota in persistently stunted young children in South India. PLoS One. 2016;11(5): e0155405.
55 Mayneris-Perxachs J, Lima AA, Guerrant RL, Leite ÁM, Moura AF, Lima NL, et al. Urinary $\mathrm{N}$-methylnicotinamide and $\beta$-aminoisobutyric acid predict catch-up growth in undernourished Brazilian children. Sci Rep. 2016;6:19780.

56 Giallourou N, Fardus-Reid F, Panic G, Veselkov K, McCormick BJJ, Olortegui MP, et al. Metabolic maturation in the first 2 years of life in resource-constrained settings and its association with postnatal growths. Sci Adv. 2020;6(15): eaay5969.

57 Zimmermann MB, Chassard C, Rohner F, N'goran EK, Nindjin C, Dostal A, et al. The effects of iron fortification on the gut microbiota in African children: a randomized controlled trial in Cote d'Ivoire. Am J Clin Nutr. 2010;92(6):1406-15.

58 Jaeggi T, Kortman GA, Moretti D, Chassard C, Holding P, Dostal A, et al. Iron fortification adversely affects the gut microbiome, increases pathogen abundance and induces intestinal inflammation in Kenyan infants. Gut. 2015;64(5):731-42.

59 McClorry S, Zavaleta N, Llanos A, Casapía M, Lönnerdal B, Slupsky $\mathrm{CM}$. Anemia in infancy is associated with alterations in systemic metabolism and microbial structure and function in a sex-specific manner: an observational study. Am J Clin Nutr. 2018;108(6): $1238-48$.

60 Armitage AE, Moretti D. The importance of iron status for young children in low- and middle-income countries: a narrative review. Pharmaceuticals. 2019;12(2):59.

61 World Health Organization. World malaria report 2016. Geneva: World Health Organization; 2016.

62 Tang M, Frank DN, Hendricks AE, Ir D, Esamai F, Liechty E, et al. Iron in micronutrient powder promotes an unfavorable gut microbiota in Kenyan infants. Nutrients. 2017;9(7):776.

63 Paganini D, Uyoga MA, Kortman GAM, Cercamondi Cl, Moretti D, Barth-Jaeggi T, et al. Prebiotic galacto-oligosaccharides mitigate the adverse effects of iron fortification on the gut microbiome: a randomised controlled study in Kenyan infants. Gut. 2017;66(11): 1956-67.

64 Dostal A, Baumgartner J, Riesen N, Chassard C, Smuts CM, Zimmermann $\mathrm{MB}$, et al. Effects of iron supplementation on dominant bacterial groups in the gut, faecal SCFA and gut inflammation: a randomised, placebo-controlled intervention trial in South African children. Br J Nutr. 2014;112(4):547-56.

65 Kalliomäki M, Collado MC, Salminen S, Isolauri E. Early differences in fecal microbiota composition in children may predict overweight. Am J Clin Nutr. 2008;87(3):534-8.

66 Luoto R, Kalliomäki M, Laitinen K, Delzenne NM, Cani PD, Salminen S, et al. Initial dietary and microbiological environments deviate in normal-weight compared to overweight children at 10 years of age. J Pediatr Gastroenterol Nutr. 2011;52(1):90-5.

67 Collado MC, Isolauri E, Laitinen K, Salminen S. Distinct composition of gut microbiota during pregnancy in overweight and normal-weight women. Am J Clin Nutr. 2008;88(4):894-9.

68 Tun HM, Bridgman SL, Chari R, Field CJ, Guttman DS, Becker AB, et al. Canadian healthy infant longitudinal development (CHILD) study investigators. Roles of birth mode and infant gut microbiota in intergenerational transmission of overweight and obesity from mother to offspring. JAMA Pediatr. 2018;172(4):368-77. 
69 Bergström A, Skov TH, Bahl MI, Roager HM, Christensen LB, Ejlerskov KT, et al. Establishment of intestinal microbiota during early life: a longitudinal, explorative study of a large cohort of Danish infants. Appl Environ Microbiol. 2014;80(9):2889-900.

70 Azad MB, Bridgman SL, Becker AB, Kozyrskyj AL. Infant antibiotic exposure and the development of childhood overweight and central adiposity. Int J Obes. 2014;38(10):1290-8.

71 Dogra S, Sakwinska O, Soh SE, Ngom-Bru C, Brück WM, Berger B, et al. Dynamics of infant gut microbiota are influenced by delivery mode and gestational duration and are associated with subsequent adiposity. mBio. 2015;6(1):e02419-14.

72 Scheepers LE, Penders J, Mbakwa CA, Thijs C, Mommers M, Arts IC. The intestinal microbiota composition and weight development in children: the koala birth cohort study. Int J Obes. 2015; 39(1):16-25.
73 White RA, Bjørnholt JV, Baird DD, Midtvedt T, Harris JR, Pagano M, et al. Novel developmental analyses identify longitudinal patterns of early gut microbiota that affect infant growth. PLoS Comput Biol. 2013;9(5):e1003042.

74 Smith-Brown P, Morrison M, Krause L, Davies PSW. Male-specific association between fat-free mass index and fecal microbiota in 2-3-year-old Australian children. J Pediatr Gastroenterol Nutr. 2018;66(1):147-51.

75 Ajslev TA, Andersen CS, Gamborg M, Sørensen TI, Jess T. Childhood overweight after establishment of the gut microbiota: the role of delivery mode, pre-pregnancy weight and early administration of antibiotics. Int J Obes. 2011;35(4):522-9.

76 Trasande L, Blustein J, Liu M, Corwin E, Cox LM, Blaser MJ. Infant antibiotic exposures and early-life body mass. Int J Obes. 2013; 37(1):16-23.

77 Gerber JS, Bryan M, Ross RK, Daymont C, Parks EP, Localio AR, et al. Antibiotic exposure during the first 6 months of life and weight gain during childhood. JAMA. 2016;315(12):1258-65. 